\title{
Treatment of Gray Hair in Vitiligo Patients by Direct Melanocytes Transplant Using Needling Micrografting and Dermabrasion Techniques
}

\author{
Khalifa E. Sharquie $^{1^{*}}$, Adil A. Noaimi ${ }^{2}$, Hana A. Al-Mudaris ${ }^{3}$ \\ ${ }^{1}$ Scientific Council of Dermatology \& Venereology, Iraqi Board for Medical Specializations, Baghdad, Iraq; ${ }^{2}$ Department of Derma- \\ tology \& Venereology, College of Medicine, University of Baghdad, Baghdad, Iraq; ${ }^{3}$ Department of Dermatology \& Venereology, \\ Baghdad Teaching Hospital, Baghdad, Iraq. \\ Email: *ksharquie@ymail.com, adilnoaimi@yahoo.com, hana_almudaris@yahoo.com
}

Received September $4^{\text {th }}, 2012$; revised October $3^{\text {rd }}, 2012$; accepted October $11^{\text {th }}, 2012$

\begin{abstract}
Background: Melanocytes transplant for treatment of vitiligo is a common therapy using different surgical procedures. But there was no interest in repigmentation of grayness of hair in the treated vitiliginous area. Objective: To do melanocytes transplant from donor area into the recipient vitiliginous area with associated gray hair. Patient and Methods: This is a case interventional study was done in Department of Dermatology/Baghdad Teaching Hospital from February 2011-March 2012. Eleven patients were enrolled in this study, six males and five females with vitiligo in association of gray hair. Their ages ranged from $8-35$ years with a mean \pm SD of $20.90 \pm 7.006$. Melanocytes transplant in patients with vitiligo using needling micrografting technique for twelve patches and direct melanocytes transplant from normal donor area into vitiliginous recipient area by dermabrasion technique for eleven patches. Dressing was applied and patients were seen every two weeks for the first month and monthly for one year. Results: Repigmentation of the vitiliginous area was started after two weeks and was obvious at one month that progressed over time. The repigmentation of hair appeared usually after few months and was obvious after four months and the repigmentation of gray hair was quicker in patients with micrografting technique than those with dermabrasion technique. The mean rate of repigmentation was $18.3 \%$ at six months and $37.5 \%$ at twelve months in micrografting technique while the mean rate of repigmentation was $9.15 \%$ at six months and $18.55 \%$ at twelve months in dermabrasion technique. Conclusions: Direct transplant of melanocytes from normal donor area into recipient vitiliginous area with associated white hair is an effective procedure to induce repigmentation of gray hair.
\end{abstract}

Keywords: Vitiligo; Melanocytes Transplants; Needling Micrograft in Vitiligo; Dermabrasion in Vitiligo; Gray Hair

\section{Introduction}

Vitiligo is a common autoimmune inflammatory disease where there is absence, deficiency or dysfunction of melanocytes [1-4].

Melanocytes transplants by different procedures in patient with vitiligo are common procedures like: punch minigraft [5-7], suction blister epidermal grafting [8], and split thickness grafting $[9,10]$ and non-cultured melanocytes grafting [11-13] and transplantation of cultured autologous melanocytes [14-16].

But still no reports appeared in the literatures showing interest in repigmentation of gray hair in the treated vitiliginous area in patient with vitiligo.

During our work doing melanocytes grafting by two techniques: the first one called melanocytes transplant in

"Corresponding author. patients with vitiligo using needling micrografting technique [17] and the second one so called direct melanocytes transplant from normal donor area into vitiliginous recipient area by dermabrasion technique [18], we noticed repigmentation of the gray hair.

So, the aim of the present work is to report eleven patients who had repigmentation of their associated gray hair.

\section{Patient and Methods}

Eleven patients with vitiligo were included in this case interventional study, six males and five females; their ages ranged from $8-35$ years with a mean \pm SD of 20.90 \pm 7.006 , the number of patches was twenty three. All clinical types of vitiligo including generalized, localized and segmental were treated (Table 1). 
Table 1. Showing the number of patients, their ages and gender, type of vitiligo, activity of the disease and number of patches.

\begin{tabular}{|c|c|c|c|c|c|}
\hline Patient no. & Age in years & Gender & Types of vitiligo & Activity of the disease & No. of patches \\
\hline 1 & 19 & $\sigma^{\pi}$ & Segmental & Stable (more than $6 \mathrm{mths}$ ) & 2 \\
\hline 2 & 22 & 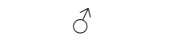 & Post herpetic localized & Stable (2 - 6 mths) & 2 \\
\hline 3 & 18 & $0^{\pi}$ & Segmental & Stable (more than $6 \mathrm{mths}$ ) & 2 \\
\hline 4 & 8 & 우 & Localized & Stable (2 - 6 mths $)$ & 3 \\
\hline 5 & 22 & 우 & Generalized & Stable (2 - 6 mths) & 1 \\
\hline 6 & 35 & 우 & Generalized & Stable (more than $6 \mathrm{mths}$ ) & 1 \\
\hline 7 & 30 & $0^{\pi}$ & Localized & Stable (more than $6 \mathrm{mths}$ ) & 2 \\
\hline 8 & 18 & $\widehat{0}$ & Localized & Stable (2 - 6 mths) & 3 \\
\hline 9 & 19 & q & Segmental & Stable (more than $6 \mathrm{mths}$ ) & 3 \\
\hline 10 & 22 & q & Segmental & Stable (more than $6 \mathrm{mths}$ ) & 1 \\
\hline 11 & 17 & $\sigma^{\lambda}$ & Generalized & Stable (2 - 6 mths) & 3 \\
\hline 11 & Mean $=20.90$ & $\widehat{\jmath}=6, q=5$ & $\begin{array}{c}\text { Generalized }=3, \\
\text { Segmental }=4, \text { Localized }=4\end{array}$ & & Sum $=23$ \\
\hline
\end{tabular}

\subsection{Inclusion Criteria}

Segmental and localized vitiligo with associated grayness of hair was included. In patient with generalized vitiligo some localized areas with gray hair were selected and treated either because are cosmetically unacceptable areas or as the patient advised. Activity of the disease was recorded for each patient to fix the duration of stability of the disease and the patients were divided into two groups stable vitiligo when there was no activity of the disease for more than 6 month before grafting and the second group when the stability of the vitiligo was ranged from 2 - 6 months only before grafting (Table 1).

\subsection{Exclusion Criteria}

Including systemic diseases like diabetes mellitus, hematological problem like bleeding tendencies, or drug that prevent clotting, immune compromised patients and other severe illnesses. All patients had stopped their treatment at least one month before grafting.

From each patient, formal consent was taken before the start the therapy, after full explanation about the nature of the disease, course, the procedure of treatment, follow up, prognosis and the need for pre and post treatment photographs. Also, the ethical approval was taken by the Scientific Committee of the Scientific Council of Dermatology \& Venereology-Iraqi Board for Medical Specializations.

Patients were photographed by a digital camera as a baseline and then every two weeks, in the same place with fixed illumination and distance by using a digital camera (Canon: power shoot G12, HS system, 10.0 mega pixels, high-sensitivity, HD movie).

\subsection{Technique of Transplant}

Direct transfer of melanocytes from normal donor area into recipient vitiliginous area with the grays of hair was carried out by two techniques:

\subsubsection{Melanocytes Transplant in Patients with Vitiligo Using Needling Micrografting Technique}

[17] which is summarized as follows: Split thickness skin graft (STSG) were harvested from different hidden normal donor areas in the body by using of ordinary surgical scalpel after injecting local anesthesia xylocain $2 \%$ with adrenalin 1:100,000. Each graft was cut by ordinary scalpel into small micro-pieces, each $1 \mathrm{~cm}^{2}$ gave about $50-80$ micro-pieces and then these micro-pieces were soaked in $0.9 \%$ normal saline until the time is ready to be grafted. Recipient area was also anaesthetized. The micropiece was taken by fine forceps and by using needle of gauge $18 \mathrm{~mm}$; the graft was implanted inside the dermis. Each piece was implanted without bothering to the surface of the graft whether dermal or epidermal and each micro-piece was about $1-5 \mathrm{~mm}$ apart from the next graft. Chemoplast glue (Spruh-pflaster, Chemo-plast Gmbh \& Co. KG, Germany) was applied over the grafted area to fix the grafted pieces and to prevent their movement and to increase the rate of graft intake. Then semi-occlusive 
plaster (Betafix, Betasan, Turkey) was used as dressing and kept for about two weeks and then removed.

\subsubsection{Direct Melanocytes Transplant from Normal Donor Area into Vitiliginous Recipient Area by Dermabrasion Technique}

[18] which could be summarized as follows: local anesthesia like xylocain was used to both the vitiliginous recipient area and the normal donor skin at the same time. Manual dermabrader was used to do superficial dermabrasion of the recipient area, and then was covered with normal saline soaked gauze to stop bleeding. Dermabrasion was also done for the donor area to remove the epidermis and papillary dermis and then the dermabraded graft was directly transferred to cover all dermabraded recipient area. Hypo-allergic semi-occlusion plaster (Betafix, Betasam, Turkey) was put as dressing on the recipient and donor areas. For both procedures systemic antibiotics were given for ten days to prevent infection. After 2 weeks, the dressing was removed and patients were evaluated every two weeks for the first month and then monthly for one year.

Table 2. Showing type of operation, sites, rate of repigmentation.

\begin{tabular}{|c|c|c|c|c|}
\hline Patient no. & Type of operation & Sites of recipient areas & $\begin{array}{l}\text { Rate of repigmentation } \\
\text { after } 6 \text { months }\end{array}$ & $\begin{array}{l}\text { Rate of repigmentation } \\
\text { after } 12 \text { months }\end{array}$ \\
\hline \multirow{2}{*}{1} & Micrograft & Chin & $40 \%$ & $60 \%$ \\
\hline & Micrograft & Lower lip & $50 \%$ & $60 \%$ \\
\hline \multirow{2}{*}{2} & Micrograft & Lower lip Rt side & $30 \%$ & $50 \%$ \\
\hline & Dermabrasion & Sterna area & $10 \%$ & $20 \%$ \\
\hline \multirow{2}{*}{3} & Micrograft & Lt cheek & $30 \%$ & $30 \%$ \\
\hline & Dermabrasion & Lt cheek & $20 \%$ & $20 \%$ \\
\hline \multirow{3}{*}{4} & Micrograft & Chest Lt side & $10 \%$ & $30 \%$ \\
\hline & Micrograft & Chest Lt side & $10 \%$ & $30 \%$ \\
\hline & Dermabrasion & Chest Lt side & $10 \%$ & $30 \%$ \\
\hline 5 & Dermabrasion & Rt lower leg & $20 \%$ & $70 \%$ \\
\hline 6 & Micrograft & Lt knee & $10 \%$ & $50 \%$ \\
\hline \multirow{2}{*}{7} & Micrograft & Lower lip Rt side & $10 \%$ & $60 \%$ \\
\hline & Micrograft & Lower lip Rt side & $15 \%$ & $50 \%$ \\
\hline \multirow{3}{*}{8} & Dermabrasion & Axilla & $10 \%$ & $20 \%$ \\
\hline & Dermabrasion & Chest & $5 \%$ & $10 \%$ \\
\hline & Dermabrasion & Rt nipple & $5 \%$ & $10 \%$ \\
\hline \multirow{3}{*}{9} & Micrograft & Abdomen Lt side & $5 \%$ & $10 \%$ \\
\hline & Micrograft & Pelvic area Lt side & $5 \%$ & $10 \%$ \\
\hline & Dermabrasion & Pelvic area Lt side & $5 \%$ & $10 \%$ \\
\hline \multirow[t]{2}{*}{10} & Micrograft & Glabrous & $5 \%$ & $10 \%$ \\
\hline & Dermabrasion & Abdomen Rt side & $5 \%$ & $5 \%$ \\
\hline \multirow[t]{2}{*}{11} & Dermabrasion & Abdomen Rt side & $5 \%$ & $5 \%$ \\
\hline & Dermabrasion & Abdomen Rt side & $5 \%$ & $5 \%$ \\
\hline \multirow{2}{*}{ Sum $=11$} & Micrograft $=12$ patches & & Mean $=18.3 \%$ & Mean $=37.5 \%$ \\
\hline & Dermabrasion $=11$ patches & & Mean $=9.1 \%$ & Mean $=18.5 \%$ \\
\hline
\end{tabular}




\section{Results}

A total of twenty two vitiligo patches with associated gray hair were treated by grafting. Micrografting technique was done for twelve patches while the dermabrasion technique was done for ten patches (Table 2).

Repigmentation of vitiligo epidermis was appeared after two weeks and was obvious at one month and the rate of repigmentation increased gradually over months. While gray hair repigmentation started usually few months after melanocytes transplant and was obvious after four months and gradually increased until we have prominent repigmentation of gray hair after six months to twelve months (Figures 1-3, Table 2). So pigmentation of gray hair started after repigmentation of vitiligo skin.
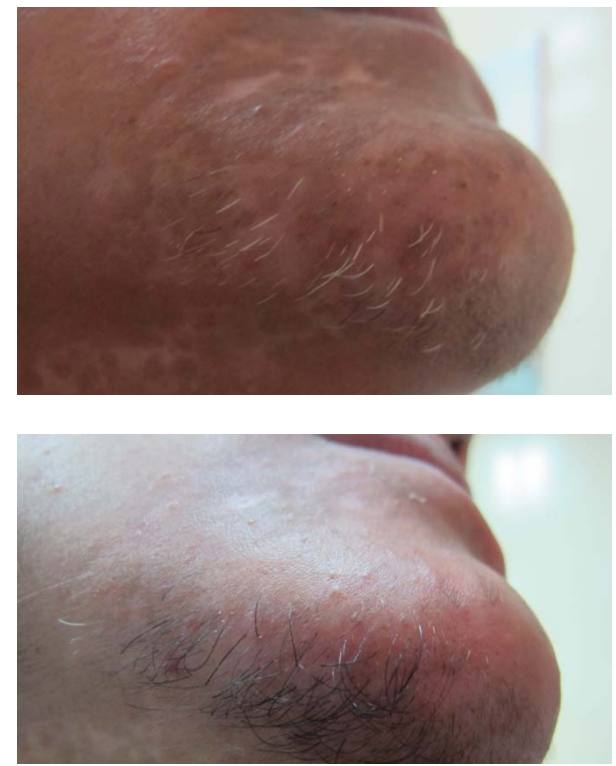

Figure 1. (a) Nineteen years old male patient with vitiligo patch associated with coarse gray hair before micrografting technique; (b) The same patient with marked repigmentation of vitiligo patch with repigmentation of gray hair after 12 months.

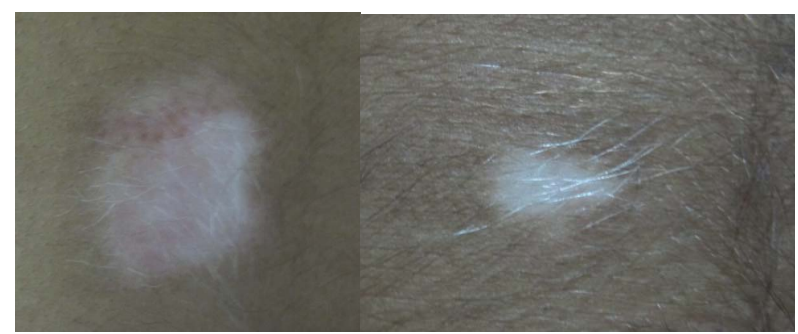

(a)

(b)

Figure 2. (a) Seventeen years old male patient with vitiligo patch on the trunk with villous gray hair one month after dermabrasion technique; (b) The same patient with marked repigmentation of vitiligo patch and gray hair 12 months after surgical therapy.

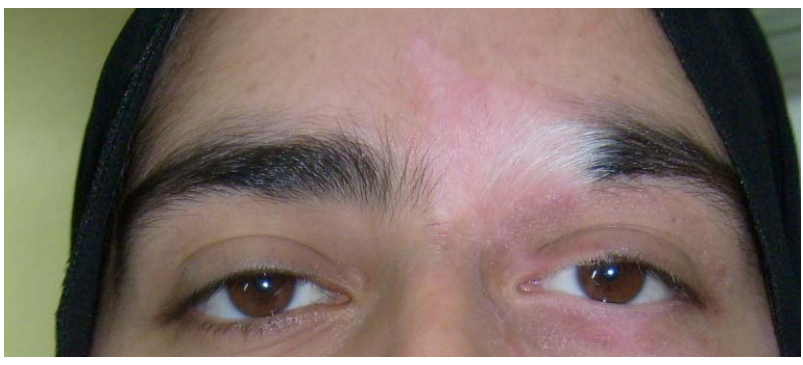

(a)

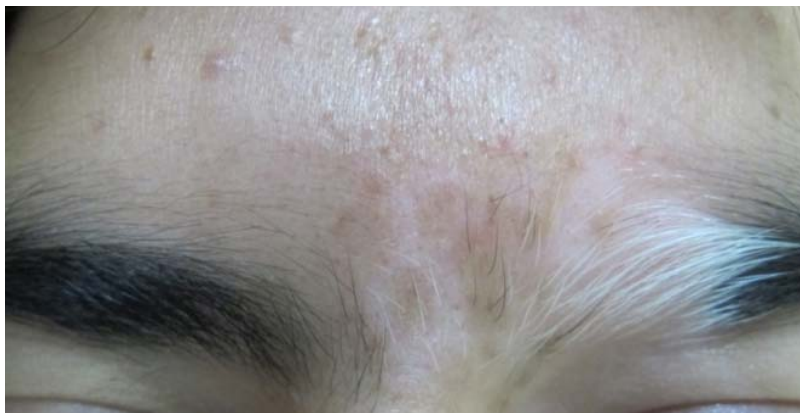

(b)

Figure 3. (a) Twenty two years old female patient with vitiligo patch associated with coarse gray hair before micrografting technique; (b) The same patient with marked repigmentation of vitiligo patch with repigmentation of gray hair after 12 months.

The results of the two techniques were comparable regarding timing of pigmentation while the rate of pigmentation was quicker and better in the micrograft technique compared with dermabrasion technique. As we have also noticed that the rate of repigmentation with micrografting technique at six months was $18.3 \%$ and at twelve months was $37.5 \%$ while with dermabrasion technique the rate of repigmentation was $9.1 \%$ at six months and $18.5 \%$ at twelve months (Figures 1-3, Table 2).

\section{Discussion}

There are many surgical procedures that have been used to treat vitiligo by autologous melanocytes transplant [416]. In the present study we did two new techniques that had already been most recently published, where direct melanocytes transfer from donor skin into vitiliginous recipient area was carried out $[17,18]$. These techniques are simple easy to do it, need no specialized personals and no sophisticated laboratories and non costly procedures.

In these techniques the rate of pigmentation was: $61.36 \%$ in patients with needling micrografting technique and $27.8 \%$ in patients with dermabrasion technique, 4 months after the time of operation $[17,18]$.

In the present works, we noticed that repigmentation of vitiligo skin started after one month while repigmenta- 
tion of gray hair was obvious after four months. This could be explained that proliferation of grafted melanocytes at the basal layer takes longer time to descend down into the gray hair follicles and then finally into the hair bulbs. This observations strongly support the hypothesis that repigmentation of gray hair originates from transplanted melanocytes. This observation has been similarly noticed in the vitiligo skin with gray hair, when treated by standard therapies for vitiligo, the repigmentation of epidermis starts first then followed by repigmentation of gray hair $[17,18]$.

We have noticed in the present study that the rate of repigmentation was quicker and better at six months and twelve months follow up with micrografting technique compared with dermabrasion procedure (Table 2).

In the micrografting technique the implantation of grafted skin was intradermally and this will raise a question how this graft will survive and how the melanocytes will migrate to the basal layer of the vitiliginous area? It is difficult to put a final answer to this difficult question. But we can speculate that the dermis of the recipient area will work as a good culture media allowing the proliferation of melanocytes. Hence the melanocytes around two weeks after transplants will migrate to the basal layer of the epidermis inducing melanogenesis and repigmentation and this is comparable to the growth of melanocytes in culture media during the process of treatment of vitiligo by culture melanocytes transplant technique [1416]. In the vitiliginous area where there is gray hair, the transplanted melanocytes will migrate either to the basal layer of the epidermis and then to the basal layer of hair follicles then to the hair matrix or the melanocytes will go directly to the basal layer of hair follicle inducing melanogenesis of gray hair.

All reports in literatures emphasizing in the importance of not using of adrenalin during anesthesia of recipient skin in order to minimize the ischemia of the grafted tissue and to increase the success rate of transplantation [5,6,8-17]. While in the present works, we used adrenalin with xylocain in all patients in the recipient area to decrease bleeding during operation. But we have noticed no effect in the rate of intake of the grafts and the process of repigmentation went normal.

In all previous grafting procedures there was no mention of repigmentation of gray hair [5-16,19-22] apart from one study by Agrawal et al. in 1995 who showed by split thickness skin graft in eight patients, repigmentation of gray hair that appeared after three months and gradually increased to have full repigmentation of gray hair after six years follow up [10,23].

These grafting procedures are indicated especially for those cases with localized grayness of hair involving the eyebrows, moustache or any area involved by grayness of hair which is not acceptable by the patient.

Further studies are highly recommended to improve these techniques in order to achieve high success rate in repigmentation of gray hair and also to treat patients with the scalp gray hair that have no skin vitiligo.

In conclusion, direct transplant of melanocytes from donor skin into recipient vitiliginous area is an easy non costly technique used to induce repigmentation of gray hair including the vitiliginous area.

\section{REFERENCES}

[1] K. E. Sharquie, "Vitiligo," Clinical and Experimental Dermatology, Vol. 9, No. 2, 1984, pp. 117-126. doi:10.1111/j.1365-2230.1984.tb00772.x

[2] K. E. Sharquie, "The Histology and Immunopathology of Vitiligo," Ph.D. Thesis, University of Sheffield, Sheffield, 1982.

[3] K. E. Sharquie, S. H. Mehenna, A. A. Naji and H. AlAzzawi, "Inflammatory Changes in Vitiligo: Stage I and II Depigmentation," The American Journal of Dermatopathology, Vol. 26, No. 2, 2004, p. 108. doi:10.1097/00000372-200404000-00004

[4] M. B. Abdel-Naser, S. K. Hann and J. C. Bystryn, "Oral Psoralen with UV-A Therapy Releases Circulating Growth Factors that Stimulates Cell Proliferation," ARCH Dermatology, Vol. 133, No. 12, 1997, pp. 1530-1533. doi:10.1001/archderm.1997.03890480050007

[5] S. Malakar and S. Dhar, "Repigmentation of Vitiligo Patches by Transplantation of Hair Follicles," International Journal of Dermatology, Vol. 38, No. 3, 1999, pp. 237-238.

[6] R. Falabella, "Surgical Therapies for Vitiligo and Other Leukoderma, Part 1: Minigrafting and Suction Epidermal Grafting," Dermatologic Therapy, Vol. 14, No. 1, 2001, pp. 7-14. doi:10.1046/j.1529-8019.2001.014001007.x

[7] K. E. Sharquie, "Pigment Minigrafting as Treatment for Dermatomal Vitiligo," The 9th Scientific Congress, College of Medicine, University of Baghdad, Baghdad, 1996.

[8] M. Ozdemir, O. Cetinkale, R. Wolf, A. Kotogyan, C. Mat, B. Tuzun and Y. Tuzun, "Comparison of Two Surgical Approaches for Treating Vitiligo: A Preliminary Study," International Journal of Dermatology, Vol. 41, No. 3, 2002, pp. 135-138. doi:10.1046/j.1365-4362.2002.01391.x

[9] A. M. Kahn and M. J. Cohen, "Treatment by Dermabrasion and Epithelial Sheet Grafting," Journal of the American Academy of Dermatology, Vol. 33, No. 4, 1995, pp. 646-648. doi:10.1016/0190-9622(95)91287-8

[10] K. Agrawal and A. Agrawal, "Repigmentation with Dermabrasion and Thin Split-Thickness Skin Graft," Dermatologic Surgery, Vol. 21, No. 4, 1995, pp. 295-300. doi:10.1016/1076-0512(94)00002-8

[11] S. V. Mulekar, "Long-Term Follow-Up Study of $142 \mathrm{~Pa}-$ tients with Vitiligo Vulgaris Treated by Autologous, NonCultured Melanocyte-Keratinocyte Cell Transplantation," International Journal of Dermatology, Vol. 44, No. 10, 2005, pp. 841-845.

doi:10.1111/j.1365-4632.2005.02226.x 
[12] S. V. Mulekar, A. Al Issa and A. Al Eisa, "Treatment of Vitiligo on Difficult-To-Treat Sites Using Autologous Noncultured Cellular Grafting," Dermatological Surgery, Vol. 35, No. 1, 2009, pp. 66-71. doi:10.1111/j.1524-4725.2008.34384.x

[13] S. V. Mulekar, "Stable Vitiligo Treated by a Combination of Low-Dose Oral Pulse Betamethasone and Autologous, Melanocyte-Keratinocyte Cell Transplantation," Dermatologic Surgery, Vol. 32, No. 4, 2006, pp. 536-541. doi:10.1111/j.1524-4725.2006.32109.x

[14] S. V. Mulekar, B. Ghwish, A. Al Issa and A. Al Eisa, "Treatment of Vitiligo Leasions by ReCell vs. Conventional Melanocyte-Keratinocyte Transplantation: Pilot Study," Journal of the European Academy of Dermatology and Venereology, Vol. 158, No. 1, 2008, pp. 45-49.

[15] B. M. El-Zawahry, N. S. Zaki, D. A. Bassiouny, R. M. Sobhi, A. Zaghloul, M. M. Khorshied and H. M. Gouda, "Atologous Melanocyte-Keratinocyte Suspension in the Treatment of Vitiligo," Journal of the European Academy of Dermatology and Venereology, Vol. 25, No. 2, 2011, pp. 215-220. doi:10.1111/j.1468-3083.2010.03759.x

[16] R. Czajkowski, W. Placer, T. Drewa, B. Kowaliszyn, J. Sir and W. Weiss, "Autologous Cultured Melanocytea in Vitiligo Treatment," Dermatologic Surgery, Vol. 33, No. 9, 2007, pp. 1027-1036. doi:10.1111/j.1524-4725.2007.33216.x

[17] K. E. Sharquie, A. A. Noaimi and H. A. Al-Mudaris, "Melanocytes Transplant in Patients with Vitiligo Using Needling Micrografting Technique," Thesis for Fellowship of Iraqi Board for Medical Specializations in Dermatology and Venereology, 2012.

[18] K. E. Sharquie, A. A. Noaimi and H. A. Al-Mudaris, "Di- rect Melanocytes Transplant from Normal Donor Area into Vitiliginous Recipient Area by Dermabrasion Technique," Journal of Cosmetic, Dermatological Sciences and Application, Vol. 2, No. 4, 2012, pp. 288-293. doi:10.4236/jedsa.2012.24055

[19] S. Sethi, B. B. Mahajan, R. R. Gupta and A. Ohri, "Comparative Evaluation of the Therapeutic Efficacy of Dermabrasion, Dermabrasion Combined with Topical 5\% 5Fluorouracil Cream, and Dermabrasion Combined with Topical Placentrex Gel in Localized Stable Vitiligo," International Journal of Dermatology, Vol. 46, No. 8, 2007, pp. 875-879. doi:10.1111/j.1365-4632.2007.03226.x

[20] N. S. Mohamed, M. F. Elgoweini and N. A. Khad, "Dermatomal Vitiligo: Therapeutic Implication of Dermabration," Journal of Pan-Arab Leaguo of Dermatologists, Vol. 19, No. 1, 2008, pp. 21-29.

[21] P. Redondo, A. G. Azcarate, L. Marques, M. G. Guzman, E. Andreu and F. Prosper, "Amniotic Membrane as a Scaffold for Melanocytes Transplantation in patients with Stable Vitiligo," Dermatology Research and Practice, 2011, pp. 1-6. doi:10.1155/2011/532139

[22] O. T. Vázquez-Martínez, H. G. Matínez-Rodríguez, L. Velásquez-Arenas, D. Baños-González, R. Ortíz-López, G. Padilla-Rivas, O. Welsh and J. Ocampo-Candiani, "Treatment of Vitiligo with a Melanocytes-Keratiocyte Cell Suspension versus Dermabrasion Only: A Pilot Study with a 12 Month Follow Up," Journal of Drugs in Dermatology, Vol. 10, No. 9, 2011, pp. 1032-1036.

[23] K. Agrawal and A. Agrawal, "Vitiligo: Surgical Repigmentation of Leukotrichia," Dermatologic Surgery, Vol. 21, No. 8, 1995, pp. 711-715. doi:10.1016/1076-0512(93)00056-I 